\title{
The Research of Tube Forming Parameter Recognition of Compensate Fuzzy Nerve Net Control
}

\author{
Ping $\mathrm{He}$ \\ Faculty of Aerospace Engineering, Shenyang Aerospace University, 110000 China \\ Izjlzjb@163.com
}

Keywords: compensate fuzzy nerve, net control, tube forming, state recognition, parameter

\begin{abstract}
The point of compensate fuzzy control is concluded. A identify model of compensate fuzzy control network is constructed. The input and output design of tube forming parameter is given, and the collection and deal of sample data is also given. Using compensate fuzzy control network does the imitate sample of model. The identify result of satisfaction is given. The new field of the tube forming parameter recognition of compensate fuzzy control network is opened.
\end{abstract}

\section{Introduction}

Selecting shrink diameter of tube forming material properties data is to state recognition, validating that proposed compensate fuzzy control is to its effectiveness. The $\mathrm{C}$ program language is used by simulation experiment. The requirements of the inputting shrink diameter tube forming state have a different pipe length of material: the wall thickness, the stitch length of discharging voltage, hardening exponent, strength coefficient, anisotropic coefficient and the elastic modulus of seven characteristics.

\section{Recognition Model of Compensate Fuzzy Control Network}

The fuzzy optimization model of tube forming factor influencing is the key problem of system development, and put all the tube forming affecting factors of power for $U=\left\{u_{1}, u_{2}, u_{n}\right\}$. Satisfy $U=$ $U_{i} \cup U_{j}$, and when I indicates whe $n i \neq j, U_{i} \cap U_{j}=\{0\}$. The among vector of tube forming affecting factors for $U=\left\{u_{i 1}, u_{i} 2, u_{i k}\right\}$. By introducing the weighted coefficient sets $A=\left(a_{1}, a_{2}, a_{n}\right), a_{i}$ present level factors $u_{i}$, that is weight size.of $u$ i. All of the collection of various factors for $V=\left\{v_{1}, v_{2},, v m\right\}$ level set [1] [4].

Based on the ideas above, for $\mathrm{r}_{\mathrm{ij}}\left(\mathrm{u} \mathrm{i}_{\mathrm{i}} \mathrm{v} \mathrm{j}\right)$,, it is introduced into dynamic variable $\mathrm{X}$ to improve, to build the fuzzy membership function of dynamic evaluation formula:of tube forming factor influencing :

when parameters $\mathrm{x}$ impact conditions no composed by parameter condition of the influence $\mathrm{x}$

$\mathrm{r}_{\mathrm{ij}}=\left\{\begin{array}{c}\mu_{\mathrm{ij}}\left(\mathrm{u}_{\mathrm{i}, \mathrm{v}}, \mathrm{x}\right) \\ 0,\end{array}\right.$

Type of dynamic variables, $\mathrm{x}$ is used to determine the different period of membership matrix. It is obtained the tube forming impact factor of the basic model of fuzzy dynamic evaluation.

$\mathrm{B}=\mathrm{A} \circ \mathrm{R}=\mathrm{b} 1 / \mathrm{v} 1+\mathrm{b} 2 / \mathrm{v} 2+\mathrm{bn} / \mathrm{vn}$

" A $\circ$ R." is said "generalized fuzzy synthetic operation," To assess for vj grade of fuzzy subsets of membership, the fuzzy B vector normalization, according to the maximum membership degree, the result of dynamic testing can be got.

In this paper, the design of compensation fuzzy network evaluation of the tube forming impact factor, to choice of aluminum alloy plates deep deformation performance parameters as input node, with tube forming factor influencing the plank as output node $\mathrm{k}$. Three input nodes is: tube bloom length, tube bloom thickness, coil length. 


\section{The Evaluation Structure of Tube Forming of Impact Factor of Compensation Fuzzy Neural Networks.}

Compensation fuzzy neural network structure has five layers: input layer, the blurred layer, compensation arithmetic, the blurred layer and output layer. It is based on fuzzy logic system between layer and layer of language variable and fuzzy if-then rules, the worst - the best operation, fuzzy reasoning method and the fuzzy functions. Among them, the each node of first layer is connected to the input vector directly; The each node of second layer represents a linguistic variable values, its role is to calculate the input vector belonging to each linguistic variable values of the fuzzy setting membership function; The each node of third layer represents a fuzzy rules, its role is to match the fuzzy rules, and calculates the suitable degree of each rule.

Fuzzy membership function for [2]

$$
\begin{aligned}
& \mu_{A i}^{k}(x)=\exp \left[-\left(\frac{x_{i}-a_{i}^{k}}{\sigma_{i}^{k}}\right)^{2}\right] \\
& \mu_{B_{i}^{k}}(y)=\exp \left[-\left(\frac{y-b^{k}}{\delta^{k}}\right)^{2}\right]
\end{aligned}
$$

The alpha with sigma is for the center and width of inputting membership functions; B with the delta is as the center and width of the output membership function.

Define input $\mathrm{X}=\left(\mathrm{x}_{1}, \mathrm{x}_{\mathrm{n}}\right)$, the theory of domain for: $\mathrm{U}=\mathrm{U}_{1} \mathrm{x} \mathrm{U}_{2} \ldots \mathrm{U}_{\mathrm{n}}, \mathrm{A}^{\prime}$ is an input fuzzy subset of theory domain in $\mathrm{U}$, according to the fuzzy rules of $\mathrm{k}, \mathrm{B}$ ' is produced in the output field of $\mathrm{V}$ fuzzy subset, the fuzzy reasoning is used in the largest algebra product of synthesis arithmetic.

As a result, the system is made up of single valued fuzzy generator, Gaussian membership function, the product of reasoning principle, negative - positive compensation algorithm and improved center of the compensation fuzzy neural networks.

In the design of tube forming impact factor of compensation fuzzy network evaluation ${ }^{[3]}$, the input node is selected of aluminum alloy tube forming deformation performance parameters, the output node is the $\mathrm{K}$ value of tube forming impact factor ${ }^{[4]}$. Three specific input nodes as follows: tube bloom length, tube bloom thickness, coil length.

\section{Simulation Examples}

Production is used frequently shrink diameterin tube bloom without die, the main influence factors include tube bloom length, tube bloom thickness, coil length, etc.

Tube bloom length is as shown in Fig. 1 for three different tube bloom length, largest diameter reduced volume and discharging voltage relationship (blank for wall thickness $1.5 \mathrm{~mm}$, diameter is the mm LD2y aluminum; discharging coil length is diameter $40 \mathrm{~mm}$ ).

Tube bloom thickness is as shown in Fig. 2 for tube bloom 1.5, 2.0 wall thickness, the relation between the largest volume andl discharging voltage, the figure shows, the biggest reducing mill with voltage is basic in a linear relationship, with the voltage increase, the biggest reducing diameter increased.

The stitch length is as discharging coil cut short, limit deformation increase. 


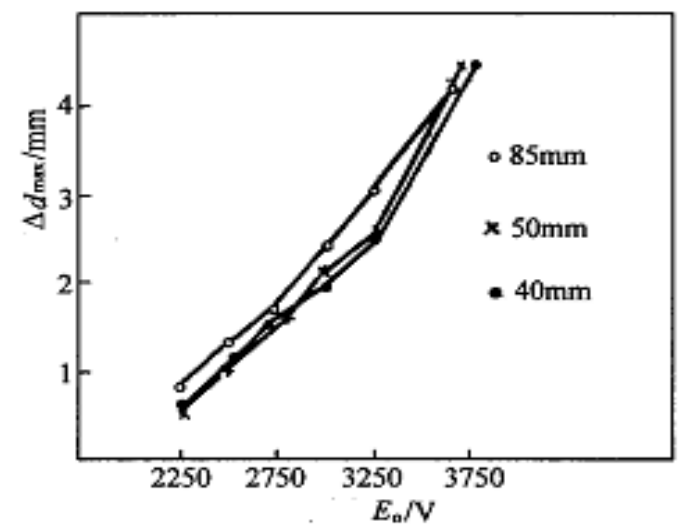

Fig. 1 Relation of different pipe length

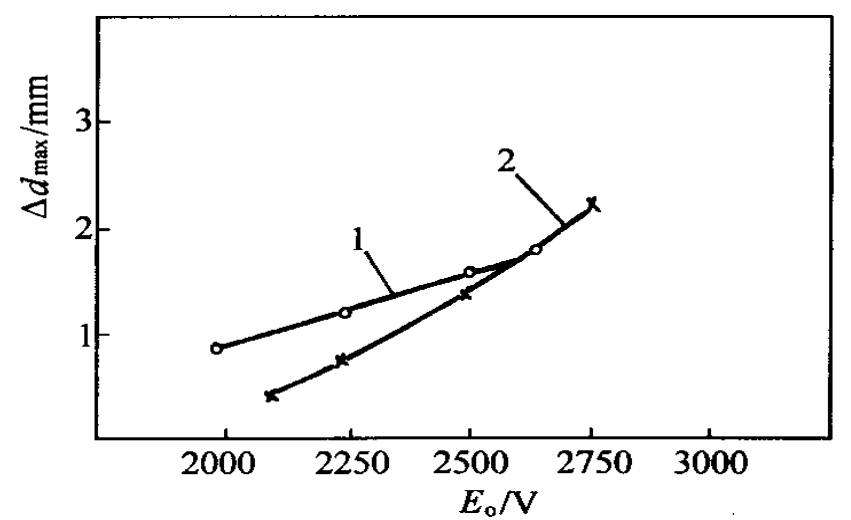

Fig. 2 Relation of different pipe thick

Application of the proposed compensation fuzzy neural networks, to the following three test which the validity of the test function optimization algorithm is to optimize operation [2].

$\mathrm{F} 1=100(\mathrm{x} 1-\mathrm{x} 2) 2+(1-\mathrm{x} 1) 2,-2.048 \leq \mathrm{xi} \leq 2.048$

$\mathrm{F} 2=4+4.5 \times 1-4 \times 2-2 \times 1 \times 2,-8 \leq \mathrm{xi} \leq 8$

$\mathrm{F} 3=0.5-[\sin (\mathrm{x} 1+\mathrm{x} 2) 1 / 2-0.5] /[1+0.001(\mathrm{x} 1+\mathrm{x} 2)] 2,-100 \leq \mathrm{xi} \leq 100$

$\mathrm{F} 1$ and $\mathrm{F} 2$ are min imum, F3is max imum.

For inspecting algorithm effectiveness, the population count for 40 , memory number 14 , immune threshold was 0.85 , the variation is 0.05 , the algorithm of probability are 20 times test.

The output is shrink diameter of three or two of the biggest diameter reduced amount, to identify its biggest diameter reduced amount. Due to the classification of the seven features used to represent a different meaning, have different dimension. Therefore, it need standardized data:

$$
x_{i j}^{\prime}=\frac{x_{i j}}{\overline{S_{j}}} ; i=1, \cdots, n ; j=1, \cdots 7
$$

Selecting 120 group experiment data. In the first experiment, and withdraw 80 group as the training data, using the suggested compensation fuzzy neural networks for the study[3], trained by establishing compensation fuzzy neural networks of complete antibody learning and accumulation. And then putting the rest of the 40 sets data as test sample[4], in order to test the recognition ability. In the experimental process, by changing the antibodies suppression threshold $p$, and studying its identification accuracy of the influence. The experimental results as shown in Fig.3 and Fig. 4.

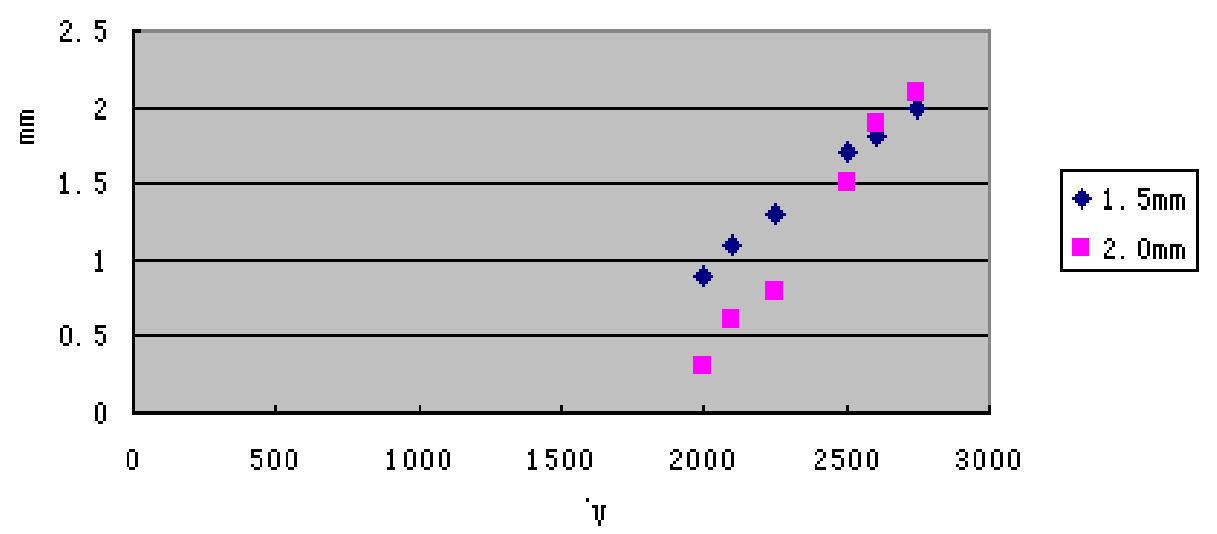

Fig. 3 curve on-line identification of most reduce diameter on different pipe thick 


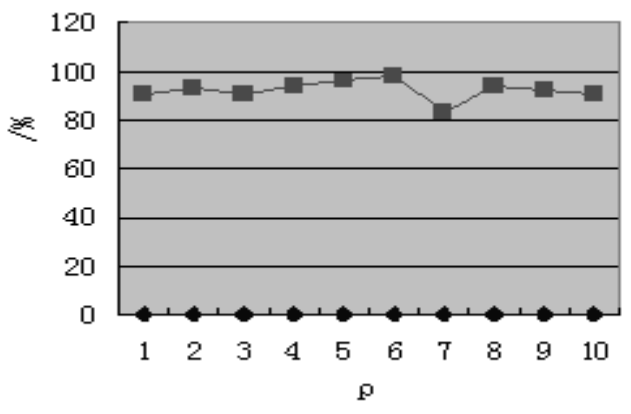

Fig. 4 Paffect on identify of pipe reduce diameter

The Fig. 3 can be seen, the algorithm is high recognition ability, average recognition rate is $94.8 \%$, which can correctly identify three states of tube shrink diameter, and identify the errors from discharging voltage fluctuations.

The simulation experiments, using the same initial population, crossover and mutation rate, is that average recognition rate is $93.2 \%$ by immune algorithm , the average recognition rate is $95.7 \%$ by compensation fuzzy neural networks. That compensation fuzzy neural networks is proposed to improve the recognition rate model of an average advantages [5] [8].

\section{Summary}

The point of compensate fuzzy control is concluded. A identify model of compensate fuzzy control network is constructed. The input and output design of tube forming parameter is given, and the collection and deal of sample data is also given. Using compensate fuzzy control network does the imitate sample of model. The identify result of satisfaction is given. The new field of the tube forming parameter recognition of compensate fuzzy control network is opened.

\section{References}

[1]. Dasgupta D, Ji z, Gonztlez F. Artificial immume system (AIS) research-m the last. five years.In the Proceedings of the International Conference On Evolutionary Computation Conference (CEC). Canbara, 2003,p.123.

[2]. Marwah G, Boggess L. Artificial immune systems for classification: some issues.In Proceedings of ICARIS 2002. Canterbury, 2002,p.149 .

[3]. T. Tröster and W. Rostek, "Advanced Hot Forming," in proceedings from The International Conference "New Development in Sheet Metal Forming Technology," Stuttgart, Germany, 2004,p.49-63.

[4]. R. Kolleck et al, "Hot Forming and Cold Forming - Two Complementary Processes for Lightweight Auto Bodies," in proceedings fromThe International Conference "New Development in Sheet Metal Forming Technology," Stuttgart, Germany, 2004 ,p. 235-244.

[5]. Dean T.A.Progress in Net-Shape Forging.Proc.of 4th Ictp.1993.

[6]. Sheljaskovs. Warm Forging in Comparison with Hot and Cold Forging.Proc.of 4th Ictp. 1993.

[7]. Schmoeckel D, Et Al. State of development and range of application of warm forging. 1992.

[8]. Schmoeckel D, et al. Warmforging as an alternative to cold extrusion and hot forging in production of large lot sizes.1993. 